\title{
Zero-order image elimination in digital holography
}

\author{
Wenwen $\mathrm{Liu}^{1}$, Xin Kang${ }^{2}$, and Xiaoyuan $\mathrm{He}^{1}$ \\ 1. Department of Engineering Mechanics, Southeast University, Nanjing, China \\ 2. Department of Engineering Mechanics, Nanjing University of Science \& Technology, Nanjing, China
}

\begin{abstract}
A bstract: A new method for eliminating zero-order image and reconstructing the original image with high quality is described in this paper. In this method, the differential of the detected hologram intensity is used instead of the hologram itself for numerical reconstruction by computing the discrete Fresnel integral. This method is based on digital image processing free of any extra optical element. It can improve the image quality significantly and give better resolution and higher accuracy of the reconstructed image. The main advantages of this method are its simplicity in experimental requirements and convenience in data processing. The theoretical analysis is given and experimental results are presented.
\end{abstract}

Key words: Digital holography, Zero-order image, Digital image processing, Fast fourier transform, Fresnel integral

\section{INTRODUCTION}

Digital holography, which uses a charged-coupled device (CCD) camera to record holograms, and then employs a computer to perform the reconstruction of the digitized holograms based on Fourier optics theory[1, 2] without chemical and physical developing, has been widely used in many areas. However, due to the low resolution of CCD sensors, the allowable angle between the object and reference beams has to be reduced to a few degrees only, so that the reconstruction image unavoidably contains the conjugate image and the zero-order image. The zero-order image includes most of the energy, thus a big bright speckle is generated in the center of the image, which causes a decrease in the resolution.

Several methods have been proposed for eliminating the zero-order diffraction image $[3,4]$, such as phase-shift technique, average intensity of the hologram elimination technique and so on. In the phase-shift technique many pieces of holograms are recorded by adjusting the phase of the reference beam, it requires to setup a rigorous experiment environment with extra phase-shift equipment to collect the holograms time after time and cannot be used to study a dynamic specimen. The method of eliminating average intensity of the hologram can wipe zero-order image simple and fast but it can only suppress part of the zero-order diffraction if the object wave intensity is not uniform.

To overcome these limitations, a new method is presented in this paper for eliminating the zero-order image in digital off-axis holography. Instead of the hologram itself, we use the differential of the hologram for reconstruction by calculating the discrete Fresnel integral. This method can simultaneously suppress the zero-order diffraction and keep the twin images intact. So, it not only can improve the image quality but also gives better resolution and higher accuracy of the reconstructed image. Furthermore, due to its simple experimental setup and its rapid process, this method has better resistance to environmental disturbances than other methods.

\section{HOLOGRAM ACQUISITION AND RECON- STRUCTION}

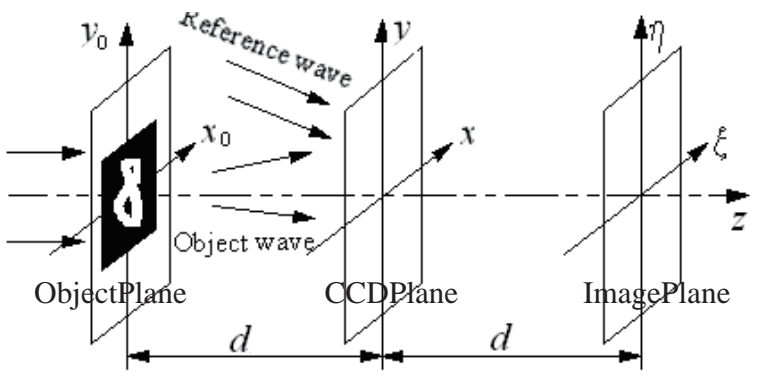

Fig. 1. Principle of digital off-axis holography.

Considering the optical path of digital off-axis holography as shown in Fig. 1 , a plane reference wave and the diffusely reflected object wave are made to interfere at the $x y$ plane, where put the CCD used to record the hologram. Under the condition of Fresnel approximation, the object wave can be reconstructed at the $O \xi \eta$ plane by computing the Fresnel integral[5] of the digitized hologram intensity $I_{H}(x, y)$,

$$
\begin{aligned}
\Psi(\xi, \eta)= & \frac{i a}{\lambda d} \exp \left[\frac{i \pi}{\lambda d}\left(\xi^{2}+\eta^{2}\right)\right] \iint R_{D}(x, y) I_{H}(x, y) \\
& \times \exp \left[\frac{i \pi}{\lambda d}\left(x^{2}+y^{2}\right)\right] \exp \left[-\frac{\pi}{\lambda d}(x \xi+y \eta)\right] d x d y
\end{aligned}
$$

Where $a$ is the amplitude of the incident wave; $d$ is the reconstruction distance, which is usually equal to the distance of object and hologram recording plane; $\lambda$ is the wavelength; $R_{D}(x, y)$ is the reconstruction reference light. The function $\Psi(\xi, \eta)$ can be digitized if the hologram transmission $I_{H}(x, y)$ is sampled on a rectangle raster of $M \times N$ matrix points, with steps $\Delta x$ and $\Delta y$ along the coordinates. Then $\xi$ and $\eta$ are replaced by $m \Delta \xi$ and $n \Delta \eta$, where $m, n$ are integers. In this case, the discrete representation of Eq.(1) is given by the following equation[6]: 


$$
\begin{aligned}
\Psi(m, n)= & \frac{i a}{\lambda d} \exp \left[\frac{i \pi}{\lambda d}\left(m^{2} \Delta \xi^{2}+n^{2} \Delta \eta^{2}\right)\right] \\
& \times F F T\left\{R_{D}(x, y) I_{H}(x, y) \exp \left[\frac{i \pi}{\lambda d}\left(k^{2} \Delta x^{2}+l^{2} \Delta y^{2}\right)\right]\right\}
\end{aligned}
$$

Where $k, l, m, n$ are integers; FFT is the fast-Fourier-transform operator; and $\Delta x$ and $\Delta y$ are the sampling intervals (the pixel size of the CCD target) in the hologram plane. The sampling intervals on the reconstructed image plane $(\Delta \xi$ and $\Delta \eta$ ) are related to the size of the CCD $(L)$ and to the distance $d$ by the relation of $\Delta \xi=\Delta \eta=\lambda d / L$. Equation 2 is an array of complex numbers, so the amplitude-contrast image and phase-contrast image can be obtained by calculating the intensity $\left|\operatorname{Re}(\Psi)^{2}+\operatorname{Im}(\Psi)^{2}\right|$ and the phase $\{\operatorname{atan}[\operatorname{Re}(\Psi) / \operatorname{Im}(\Psi)]\}$ of $\Psi(m, n)$, respectively.

\section{SPATIAL SPECTRUM OF DIGITAL OFF-AXIS HOLOGRAM}

In the hologram plane oxy the interference between the object wave $O$ and the plane reference wave $R$ produces a distribution of intensity, which is generally written as a sum of four terms,

$$
I_{H}(x, y)=I_{R}+I_{O}(x . y)+R^{*} O+R O^{*}
$$

where $I_{R}$ is the intensity of the reference wave and $I_{O}(x, y)$ is the intensity of the object wave, $R^{*} O$ and $R O^{*}$ are the interference terms, and $R^{*}$ and $O^{*}$ are the complex conjugates of the two waves. Let us assume that the hologram reconstruction is performed by illumination with a plane wave $U$. Then, as in classical holography, the reconstructed wave front in the hologram plane is given by

$$
\Psi(x, y)=U I_{R}+U I_{O}(x . y)+U R^{*} O+U R O^{*}
$$

The first two terms of Eq. (4) form the zero-order diffraction. The third and the fourth term generate the twin images of the specimen. The third term $U R^{*} O$ produces a virtual image located at the position initially occupied by the specimen, and the fourth term $U R O^{*}$ produces a real image located on the other side of the hologram. In off-axis holography the object wave $O$ and the reference wave $R$ arrive in the hologram plane with separated directions. If we assume that a reference wave with obliquity of $\theta$ is in the form of $R(x, y)=\sqrt{I_{R}} \exp \left(i k_{0} x\right)$, where $k_{0}=2 \pi \sin \theta / \lambda$, the hologram intensity becomes

$$
\begin{aligned}
I_{H}(x, y)= & I_{R}+I_{O}(x . y)+\sqrt{I_{R}} \exp \left(-i k_{0} x\right) O \\
& +\sqrt{I_{R}} \exp \left(i k_{0} x\right) O^{*}
\end{aligned}
$$

Performing a Fourier transformation on both sides of Eq.(5), we have

$$
\begin{aligned}
\tilde{I}_{H}\left(f_{x}, f_{y}\right) & =\tilde{I}_{R}+\tilde{I}_{O}\left(f_{x}, f_{y}\right)+\sqrt{I_{R}} \tilde{O}\left(f_{x}-k_{0}, f_{y}\right) \\
& +\sqrt{I_{R}} \tilde{O}^{*}\left(f_{x}+k_{0}, f_{y}\right)
\end{aligned}
$$

Digital hologram after FFT transform is shown in Fig. 2(a). We can see clearly that there are three parts in the picture, the spatial frequencies corresponding to the zero-order image are located in the center of it, the other two parts are the spatial frequencies corresponding to the conjugate image and real image. Its one dimension condition is shown in Fig. 2(b), the first term is a $\delta$ function located at the origin of the spatial-frequency plane. The second term, being proportional to the autocorrelation function of $\tilde{O}\left(f_{x}, f_{y}\right)$, is centered on the origin too, with twice the extent of the object wave spectrum. The third term is the object wave spectrum shifted upwards a distance $k_{0}$, while the fourth term is the conjugate of the object wave spectrum, shifted downwards the same distance $k_{0}$.

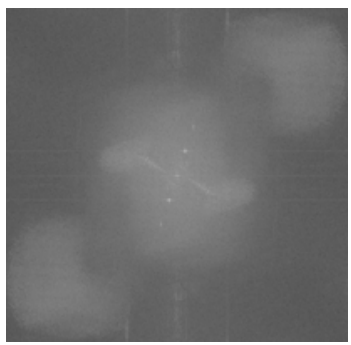

(a)

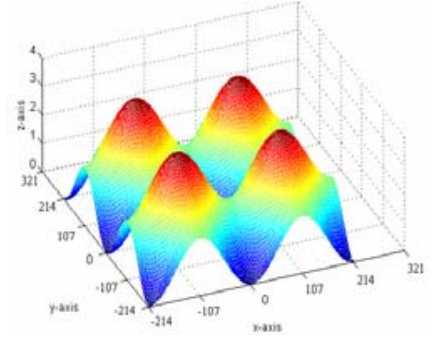

(c)

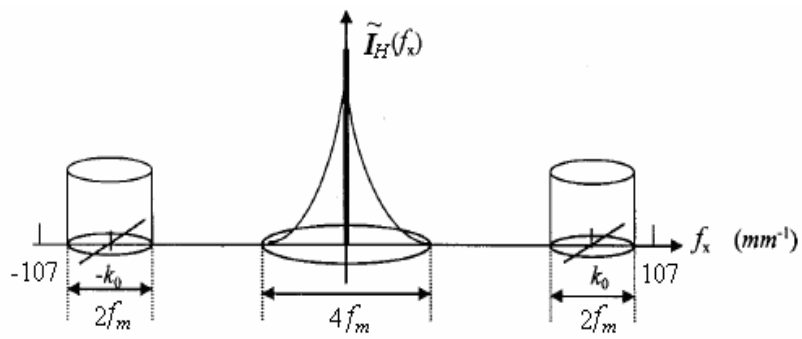

(b)

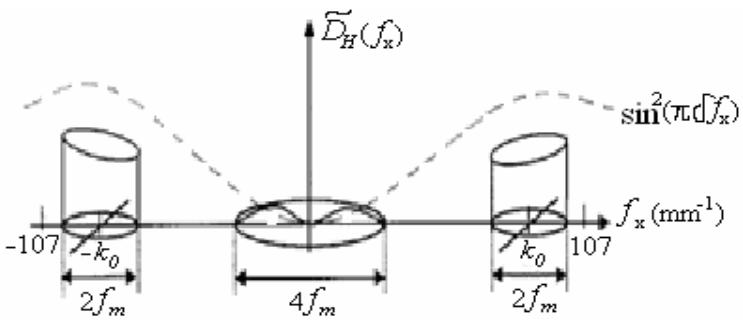

(d)

Fig. 2. Schematic diagrams of differential dispose of the hologram intensity: (a) Digital hologram after FFT transform, (b) Schematic of the spatial spectrum of the digital hologram, (c) 3-dimension schematic diagram of $K\left(f_{x}, f_{y}\right)$, (d) 1-dimension schematic diagram of the spatial spectrum of the disposed digital hologram intensity. 
Since it is necessary to have at least two sampled points for each fringe in a holographic recording, the maximum spatial frequency of a hologram is $f_{\max }=1 / 2 \Delta$ when a CCD with sensors spaced by $\Delta$ is used for hologram recording. For $\Delta=4.65 \mu \mathrm{m}$ (the actual spacing for the CCD sensors used in this work), $f_{\text {max }}$ is about 107 $\mathrm{mm}^{-1}$. It can be seen from Fig. 2(b) that the maximum spectral width of the object wave spectrum is only 53 $\mathrm{mm}^{-1}$.

\section{DIFFERENTIAL OF DIGITAL OFF-AXIS HOLOGRAM}

The differential of the hologram intensity is defined as

$$
\begin{aligned}
D_{H}(x, y) & =\left(\frac{\partial I_{H}(x, y)}{\partial x}\right)+\left(\frac{\partial I_{H}(x, y)}{\partial y}\right) \\
& =\frac{I_{H}(x+\delta, y)+I_{H}(x, y+\delta)-2 I_{H}(x, y)}{\delta}
\end{aligned}
$$

where $\delta$ is the distance between neighboring pixels.

Performing a Fourier transformation on both sides of Eq.(7) to consider the spectrum of digital processed hologram, we have

$$
\operatorname{DFT}\left[D_{H}(x, y)\right]=\frac{\tilde{I}_{H}\left(f_{x}, f_{y}\right)\left[\exp \left(i 2 \pi \delta f_{x}\right)+\exp \left(i 2 \pi \delta f_{y}\right)-2\right]}{\delta}
$$

where $\hat{I}_{H}\left(f_{x}, f_{y}\right)$ is the Fourier transform of $I_{H}(x, y)$. Considering real part of Eq.(8), we have

$$
\begin{aligned}
\operatorname{DFT}\left[D_{H}(x, y)\right]_{\text {real }} & =\tilde{D}_{H}\left(f_{x}, f_{y}\right)_{\text {real }} \\
& =\frac{\tilde{I}_{H}\left(f_{x}, f_{y}\right)\left[\cos \left(2 \pi \delta f_{x}\right)+\cos \left(2 \pi \delta f_{y}\right)-2\right]}{\delta} \\
& =\frac{\tilde{I}_{H}\left(f_{x}, f_{y}\right)\left[2 \sin ^{2}\left(\pi \delta f_{x}\right)+2 \sin ^{2}\left(\pi \delta f_{y}\right)\right]}{\delta} \\
& \doteq \tilde{I}_{H}\left(f_{x}, f_{y}\right) \times K\left(f_{x}, f_{y}\right)
\end{aligned}
$$

Figure 2(c) presents the 3-dimension schematic diagram of $K\left(f_{x}, f_{y}\right)$. Due to the separability of this equation, we need only investigate the one dimensional spectrum $\check{D}_{H}\left(f_{x}\right)_{\text {real }}$, which is shown schematically in Fig. 2(d). We can see that the spectrum of the zero-order diffraction is efficiently suppressed by $\sin ^{2}\left(\pi \delta f_{x}\right)$ function, which is figured by broken line, and the spectra of the twin images are almost intact except for a small change in intensity. Therefore, the zero-order diffraction will almost disappear from the reconstructed image, and the quality of the reconstructed twin images will be significantly improved when the differential of the detected hologram is used for reconstruction instead of the hologram itself.

It can be seen in Fig. 2(d) that the suppression of the spectrum of zero-order diffraction is accompanied by a modification in the spectrum of two twin images. This means that the reconstructed twin images will be modified to some extent when this method is used. However, as mentioned above, the maximum spectral width of the twin image is only $53 \mathrm{~mm}^{-1}$, which is much smaller than the period of the function $\sin ^{2}\left(\pi \delta f_{x}\right)$. The spectra of the twin images undergo no substantial change when multiplied by the function $\sin ^{2}\left(\pi \delta f_{x}\right)$, except for a small change in their intensity. On other hand, $\sin ^{2}\left(\pi \delta f_{x}\right)$ only slightly changes the magnitude of the spectra of the twin images by simultaneously weakening their lower frequency components and strengthening their higher frequency components. According to Fourier imaging principles, this kind of modification only results in an alteration of the image contrast. So, when we reconstruct a hologram with this method, we do slightly alter the image quality of the twin images. But in comparison with the significant improvement in image quality due to the elimination of the zero-order diffraction, this alteration is practically imperceptible.

\section{EXPERIMENTAL RESULTS}

To verify the efficiency of this method, a hologram was reconstructed with and without the elimination of the zero-order diffraction.

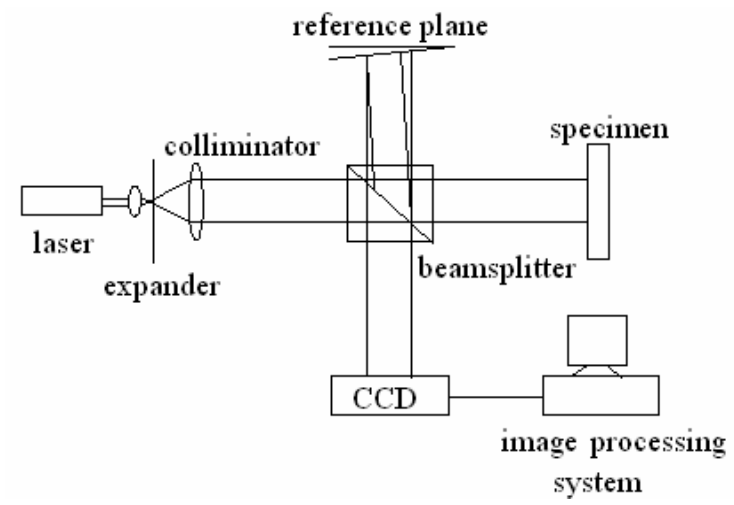

Fig.3. Experimental setup.

The experimental setup is shown in Fig. 3, with a dice as specimen. A He-Ne laser is used as the light source, with wavelength of $632.8 \mathrm{~nm}$. The laser, linearly polarized plane wave fronts are produced by a beam expander Be including a pinhole for spatial filtering. After get through a beamsplitter, the wave fronts become two beams, one illuminates the specimen to be tested, the other illuminates the reference plane. Then the two beams both come back to the beamsplitter respectively after reflecting, and interfere at the target of a CCD camera to form the hologram, which is shown in Fig. 4(a).

The distance between the CCD camera and the specimen is $40 \mathrm{~cm}$. The image in Fig. 4(a) contains 1024 *1024 pixels, and the area of the sensitive chip of the CCD is $4.65 * 4.65 \mu \mathrm{m}$. The interference fringes characterizing the off-axis geometry are clearly observable in this image. Figure 4(b) shows the amplitude-contrast image obtained by numerical reconstruction of the original hologram shown in Fig. 4(a). Figure 4(c) shows the differential of the original hologram. Figure 4(d) presents the amplitude-contrast image obtained by numerical reconstruction of the differential of the hologram. It is obvious that the zero-order diffraction has been efficiently eliminated especially in the first quadrant where the real image is located in, and the resolution of the reconstructed image is significantly improved.

Because only one frame of the digitized hologram is required in this method and the experimental procedures involve no troublesome processing, the data acquisition is very fast in this experiment. Furthermore, the differential is a classic tool for digital image processing, and there are 


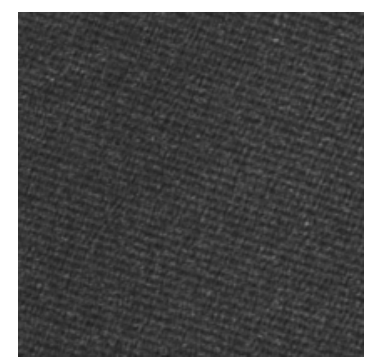

(a)

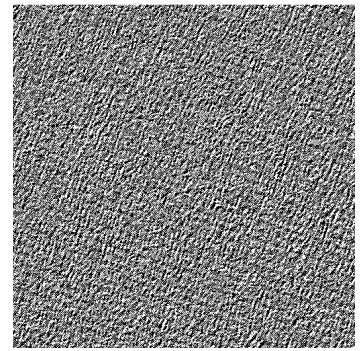

(c)

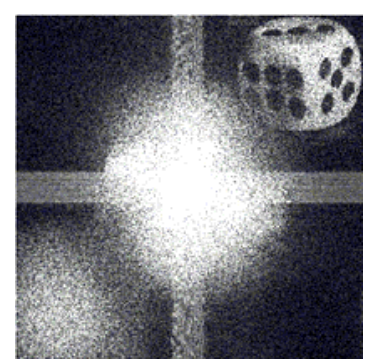

(b)

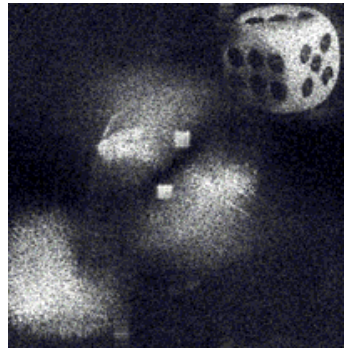

(d)
Fig. 4. Elimination of the zero order of diffraction in digital holography: (a) original off-axis hologram, (b) amplitude-contrast image obtained by numerical reconstruction of the original hologram, (c) the disposed hologram, (d) amplitude-contrast image obtained by numerical reconstruction of the differential of the original hologram.

many standard computer programs for its computation. So eliminating the zero-order diffraction with this method is much more convenient and faster than with other methods.

\section{CONCLUSION}

A simple method has been developed for eliminating the zero-order diffraction in digital holography. In this method, the differential of the hologram is used instead of the detected hologram itself for reconstruction by computing the Fresnel integral. By theory and experiment analysis, it is proved that the zero-order diffraction has been efficiently eliminated especially in the first quadrant where the real image is located in. This method is based on digital image processing free of any extra optical element, so the method has good experimental application value. The application of this method not only results in an improvement in the image quality but also produces better resolution and higher accuracy in the reconstructed twin images.

\section{ACKNOWLEDGMENTS}

Funding from project 10472026 supported by National Natural Science Foundation of China, project 2006CB300404 supported by National basis research program of China and project BK2006102 supported by Province Natural Science Foundation of Jiangsu are gratefully acknowledged.

\section{REFERENCES}

1. Cuche E., Marquet P. and Cepeursinge C., J. Appl. Opt., 39-23 (2000), pp. 4070-4075.

2. Shedin S., Pedrini G. and Tiziani H J., J. Appl. Opt., 40-1(2001), pp. 100-103.

3. Takaki Y., Kawai H. and Ohzu H., J. Appl. Opt., 38-23(1999), pp. 4990-4996.

4. S. Pasko and R. Jozwicki, J. Opto-electronics Review, 11-3(2003), pp. 203-209.

5. Goodman J. W., Introduction to Fourier optics, McGraw-Hill, NewYork(1996), pp. 64-80.

6. E.Cuche, F.Bevilacqua, and C.Depeursinge, J. Opt. Lett. 24-5(1999), pp. 291-293. 